\title{
Using Solar Energy for Charging Electric Vehicles in Poland - a case study
}

\author{
Witold Marańda ${ }^{1, *}$ \\ ${ }^{1}$ Department of Microelectronics and Computer Science, Lodz University of Technology, \\ ul. Wólczańska 221/223, 90-924 Łódź, Poland
}

\begin{abstract}
The recent advancements in the fields of electric propulsion and battery technology have made possible the implementation of all-electric transport within the coming decades. However, the widespread use of electric cars could seriously threaten the existing capabilities of energy generation and the load of the utility grid. This work investigates the use of solar energy for producing energy for transportation locally, using energy buffering and minimizing grid energy transfers. A case-study of an electric car and dedicated $\mathrm{PV}$-system is investigated for the duration of a full year in Polish climatic conditions.
\end{abstract}

\section{Introduction}

Electric propulsion has undeniable superiority over combustion engine in nearly all aspects. The recent progress in battery technology, namely Li-ion and supercapacitors, has brought the perspective of all-electric transport very close to reality. Although the commercially available electric vehicles (EV) are still not affordable for average customers, many countries are introducing incentives to foster the market transformation with expectations to achieve the target of all-electric transport within 1-2 decades.

This process, however, might be seriously delayed or threatened by the shortage of electric energy generation capabilities and excessive transfers over existing utility network. The total Net Calorific Value of all fuel (gasoline, diesel and LPG) consumed by vehicles in Poland in 2016 [1, 2] amounts to nearly $240 \mathrm{TWh} /$ year, which is higher than volume of electricity production in $2016-162 \mathrm{TWh} /$ year [3]. Even if only the passenger cars with efficient electric engines are considered, those alone would still need about $35 \mathrm{TWh} /$ year. Therefore, the energy that is now taken from oil must be found elsewhere. Nowadays, this supplementary energy generation can come from renewable energy sources (RES). The key obstacle for large-scale adoption of RES is the negative impact of excessive energy transfers on energy distribution network [4].

EV are considered the key element for successful integration of RES with electricity grid. Their key role would be to absorb the excess of renewable energy, that is highly fluctuating and to some degree unpredictable. The major research towards EV deployment strategies is to develop smart charge-plans for EV, taking into accounts drivers habits, economic factors and electrical grid limitations [5].

\footnotetext{
*Corresponding author: maranda@dmcs.p.lodz.pl
} 
The previous research focused mainly of large scale deployment scenarios [6] (PVpowered parking lots, fleet management for transportation companies) or adjusting EV charge-plans for residential PV-systems [7].

This work, in contrast, focuses on investigating the balance of energy transfers and adjustment of a dedicated PV-system for charging an electric car throughout the year in Polish climatic conditions, but without dealing with present economic details. The key idea is that energy for transportation purpose should be produced locally and grid energy transfers should be minimized. The investigated scenario is simplified, but realistic.

The presented method of finding energy-neutrality is universal and applicable for any climate, but the quantitative results in this paper have been calculated for Polish solar irradiance data.

\section{Investigated scenario}

The use-case scenario assumes an extensive use of electric car every day and powering it primarily with locally produced electricity from a PV-system. The simulation range covers the data for two currently available EVs and typical roof-size PV-systems.

The key assumptions for the investigated setup are as follows:

- $\quad$ EV is used daily and battery charging to the full capacity is needed every night.

- Electric energy is accumulated in a local buffer during the day, primarily from the PV-system and - if needed - also from the utility grid, while excess of energy is fed to the grid.

- Energy accumulation and vehicle charging are separated in time. Charging process is transparent to the utility grid, since it takes place solely from the local energy buffer.

- Analysis is done in terms of transfers of energy quantities and efficiencies of energy transfers are assumed ideal.

The results from the investigated system might be interpreted as boundary conditions for more realistic situations where electric cars are used less extensively. Moreover, in this scenario the excess of produced energy is always fed to the grid, whereas in practice other local loads may exist and be powered with this energy. This might further improve the results, but this is not investigated here in order to keep the results interpretation simple.

\subsection{Components}

The investigated system, shown in Fig. 1, consists of four components:

- Photovoltaic system (PV) delivering power with varying rate during the day and with high seasonal differences; not suitable for direct vehicle charging. The basic parameter is the annual energy yield (which corresponds to rated power in $\mathrm{kWp}$ or area size in $\mathrm{m}^{2}$ ).

- Energy buffer (B) used for local energy buffering with the day-to-nigh operation principle; implemented with supercapacitors or Li-ion cells; EV charging is done directly from this buffer.

- $\quad$ EV (V) represented in this system as the Li-ion battery needed to be charged for everyday operation. Table 1 show basic parameters of two available on market EVs, which will be used as reference points in the results.

- Utility grid $(G)$, which serves as auxiliary energy source to charge the local buffer or sink the excess of energy from PV, depending on the season of the year. 


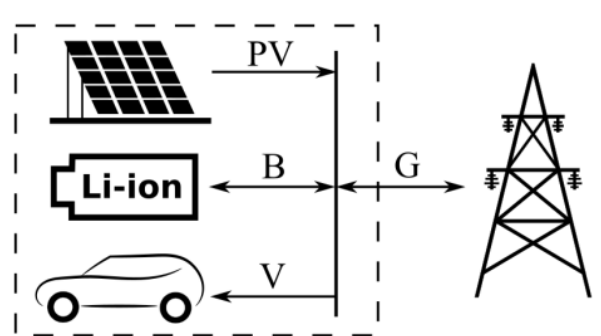

Fig. 1. Components of the investigated system.

Table 1. Electric cars data.

\begin{tabular}{|c|c|c|c|c|}
\hline Vehicle & $\begin{array}{c}\text { Battery } \\
\text { Capacity } \\
{[\mathbf{k W h}]}\end{array}$ & $\begin{array}{c}\text { Daily range } \\
\text { (average) } \\
{[\mathbf{k m}]}\end{array}$ & $\begin{array}{c}\text { Annual } \\
\text { consumption } \\
{[\mathbf{M W h}]}\end{array}$ & $\begin{array}{c}\text { Annual } \\
\text { Millage } \\
{[\mathbf{k m}]}\end{array}$ \\
\hline Mitsubishi iMiEV & 16 & $\sim 100$ & 5.8 & $\sim 35.000$ \\
\hline Nissan Leaf & 30 & $\sim 150$ & 10.9 & $\sim 60.000$ \\
\hline
\end{tabular}

\subsection{Operation principles}

System operation follows the following rules:

- Energy accumulation (in local buffer B) is spread over the day with low power intensity, using primarily PV and grid G as auxiliary source,

- Vehicle charging is performed with high power rate during the night hours, charging is done from the local buffer B only, and no energy is taken from the grid G

- Energy accumulation and charging processes are separated in time.

System operation can be visualized by the energy management diagram, shown in Fig. 2, where states corresponds to the energy flows between components, using the notation (source $\rightarrow$ sink) as proposed in [8]. Here, the PV can only be the source, V is always the sink, whereas $\mathrm{B}$ and $\mathrm{G}$ can play both roles at different times. The empty state $(\rightarrow)$ indicates the idleness of the components.

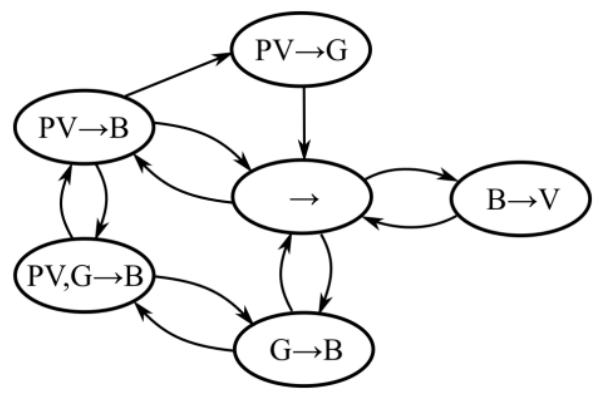

Fig. 2. Energy management diagram for the investigated system. 


\section{System analysis}

\subsection{Energy transfers}

Energy transfer analysis is performed on a yearly basis. The symbols use in the formula and the graphs have the following meaning:

- $\quad E_{P V}-$ annual energy yield from PV-system; this can be easily estimated for any PV-array size and system location using, for example, online services as [9].

- $\quad E_{V}$ - annual energy (electricity) consumption of an electric car; this can be easily translated into an annual mileage of the vehicle using its specification details, as shown in Table 1.

- $\quad E_{F}$ (and $E_{G}$ ) - annual amounts of energy fed to (or taken from) the grid

The system operation can be divided into separate daily cycles, where charging of EV must be full accomplished. Therefore, the size of battery B is equal to the capacity of car battery and defined as:

$$
E_{B}=E_{V} / 365
$$

The daily system operation can follow one of three general cases, presented in Fig. 3-5, where the relative proportions between areas are preserved. The daily energy quantities are denoted by an i-index, so $E_{P V}^{i}, E_{V}^{i}, E_{F}^{i}, E_{G}^{i}$ refer to energy during $i$-th day within a year.

Fig. 3 shows and ideal case where energy production from PV matches the vehicle charge demand and no energy transfer to-or-from the grid is needed. The PV-power peak may be low, but its time interval is wider than charging profile.

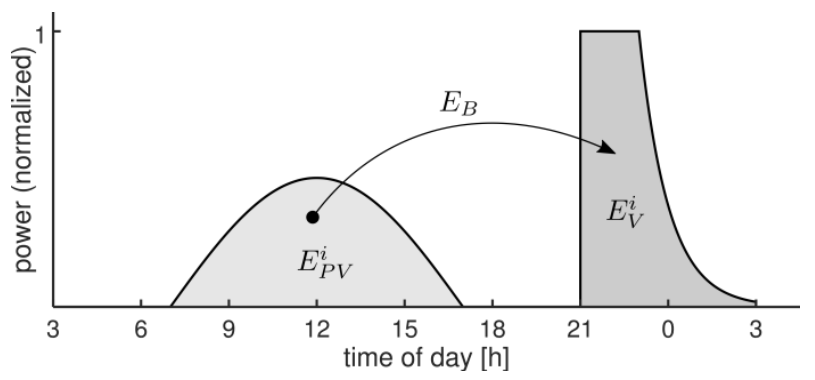

Fig. 3. Ideal case of energy transfer from PV-system (via local buffer) to car battery.

In practice, the will always be either a daily surplus or shortage of PV-energy, so transfers to-or-from the grid will take place, as shown in Fig. 4 and 5.

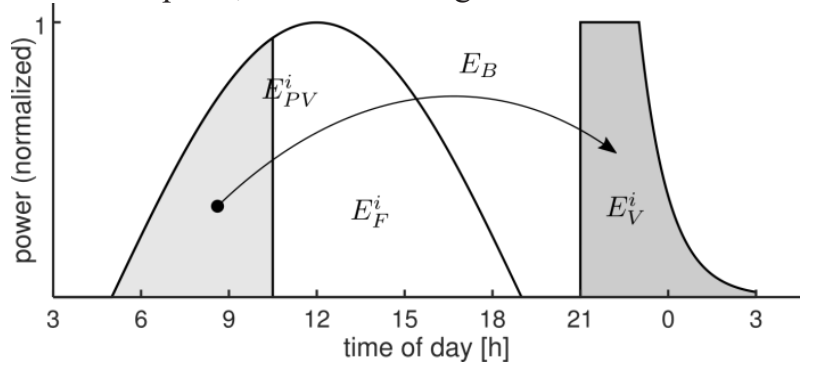

Fig. 4. Daily cycle with surplus of energy from PV-system (sunny day). 


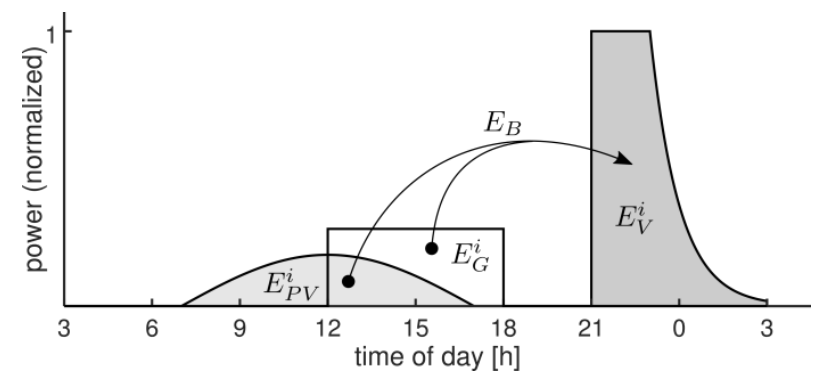

Fig. 5. Daily cycle with shortage of energy from PV-system (cloudy day).

In case where auxiliary energy must be taken from the grid (as in Fig. 5), the several strategies for energy accumulation can be implemented - here the grid consumption is activated at midday based on the half-day anticipation of PV output (other strategies are also possible), but actual shapes of $E_{G}^{i}$ have no influence on energy balance analysis.

Obviously, the following equations hold:

$$
\begin{aligned}
& \sum_{i=1}^{365} E_{P V}^{i}=E_{P V} \\
& \sum_{i=1}^{365} E_{V}^{i}=E_{V} \\
& \sum_{i=\text { day }}^{\text {sunn }} E_{F}^{i}=E_{F} \\
& \sum_{i=\text { day }}^{\text {cloud }} E_{G}^{i}=E_{G} \\
& E_{B}=E_{V}^{i}=\text { conts. }
\end{aligned}
$$

\subsection{Grid energy balance and grid energy neutrality}

Studying particular energy transfers allows also to find the conditions for energy neutrality of the system regarding its grid interactions. The energy neutrality is defined here as the equal balance between production to- and consumption from the grid, over the whole examined period:

$$
\Delta E=E_{F}-E_{G}=0
$$

The energy neutrality is important factor for the utility network, especially if some seasonal energy buffering might be implemented on a global scale. Obviously, energy neutrality may be achieved with extremely different absolute values of $E_{F}$ and $E_{G}$, but still yielding the balance $\Delta E=0$.

It can be demonstrated that grid energy balance depends solely on difference between annul PV production and annual vehicle consumption, regardless of any daily cycles details. From Fig. 4 and 5 and equations (2-5) it follows:

$$
\begin{aligned}
E_{F}-E_{G}= & \sum_{i=\text { day }}^{\text {sunny }}\left(E_{P V}^{i}-E_{V}^{i}\right)-\sum_{i=d a y}^{\text {cloudy }}\left(E_{V}^{i}-E_{P V}^{i}\right)= \\
= & \sum_{i=d a y}^{\text {sunny }} E_{P V}^{i}+\sum_{i=d a y}^{\text {cloudy }} E_{P V}^{i}-\sum_{i=d a y}^{\text {sunny }} E_{V}^{i}-\sum_{i=\text { day }}^{\text {cloudy }} E_{V}^{i}= \\
& =E_{P V}-E_{V}
\end{aligned}
$$

Therefore, the energy neutrality can be achieved when PV production matches the vehicle demand on a yearly basis: 


$$
\Delta E=0 \Leftrightarrow E_{P V}=E_{V}
$$

It can be argued that even if daily cycles are less regular (e.g. charging takes place every few days or charging cycles are of various depth), the relation (7) still holds to the large extent.

\section{Simulation results}

\subsection{Goals and data}

The numerical simulation focuses on finding annual grid energy transfers $E_{F}$ and $E_{G}$ in 2-dimensional space of $E_{P V}$ and $E_{V}$. The results may be used as recommendations for choosing optimal size of PV-system for the EV annual millage.

Analysis has been performed for the full year 2017. The solar energy data have been collected with a calibrated irradiance sensor in $5 \mathrm{~s}$ samples, at Solar Laboratory of Dept. of Microelectronics and Computer Science, Lodz University of Technology (Poland). The computation of PV-array output has been simulated taking into account typical PV efficiency and ambient temperature influence, as described in [10].

\subsection{Grid energy balance}

The numerical computations confirm the relation given by equation (7), that grid interaction (Fig. 6) is strictly equal to balance between local production $\left(E_{P V}\right)$ and consumption $\left(E_{V}\right)$.

The energy neutrality is reached for exact match between annual PV yield and vehicle consumption. This relation may be a starting point to further economic analysis if grid feedin and purchase tariffs are taken into account.

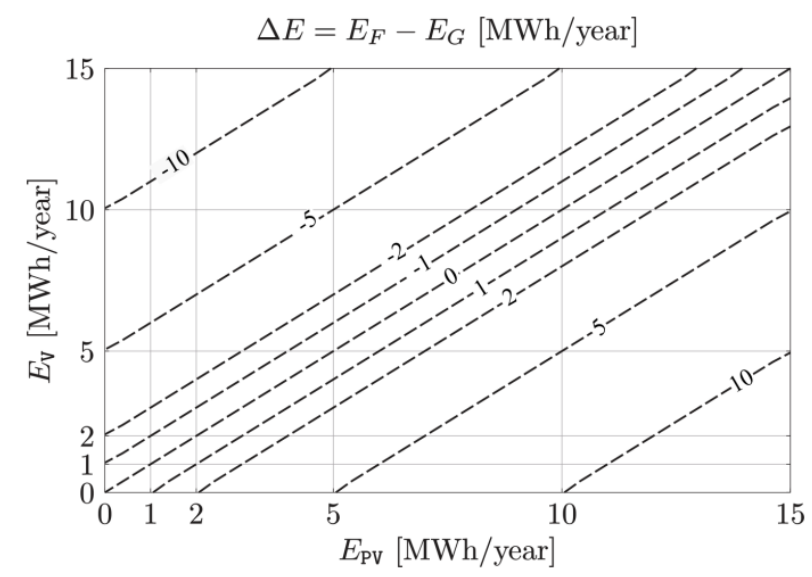

Fig. 6. Annual energy balance for the investigated system.

\subsection{Grid energy transfers}

In order to fully understand the behaviour of the investigated system, the energy balance must be decomposed into separate transfers to the grid $\left(E_{F}\right)$ an from the grid $\left(E_{G}\right)$, as in Fig. 7 and Fig. 8, showing absolute annual amounts of energy. 


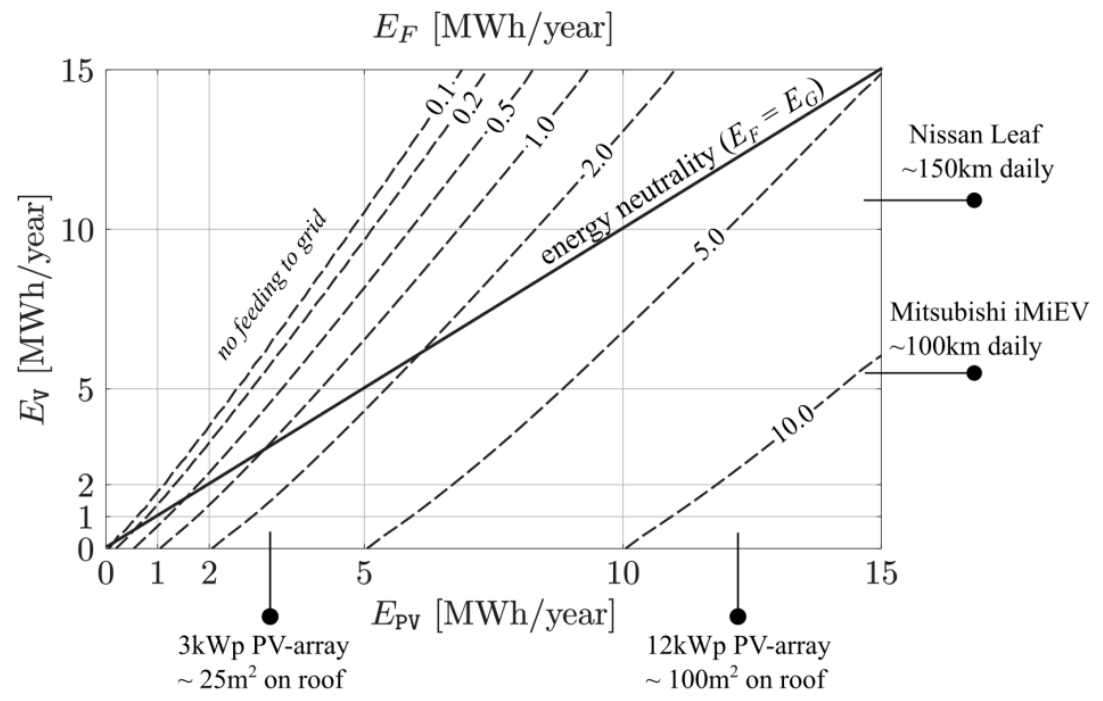

Fig. 7. Surplus of energy fed to the grid (annually).

It turns out, that even a small PV-system of $3 \mathrm{kWp}$ (that could be easily accommodated on most family-house roofs) would be sufficient to supply Mitsubishi iMiEV with the additional purchase of less than $3 \mathrm{MWh}$ extra energy per year, but with virtually no feed to the grid. For energy-neutrality, the PV-aray size should be around $6 \mathrm{kWp}$ (which is still acceptably small for private ownership) and the generated grid transfers would be as small as $\pm 2 \mathrm{MWh}$ per year.

$E_{G}[\mathrm{MWh} /$ year $]$

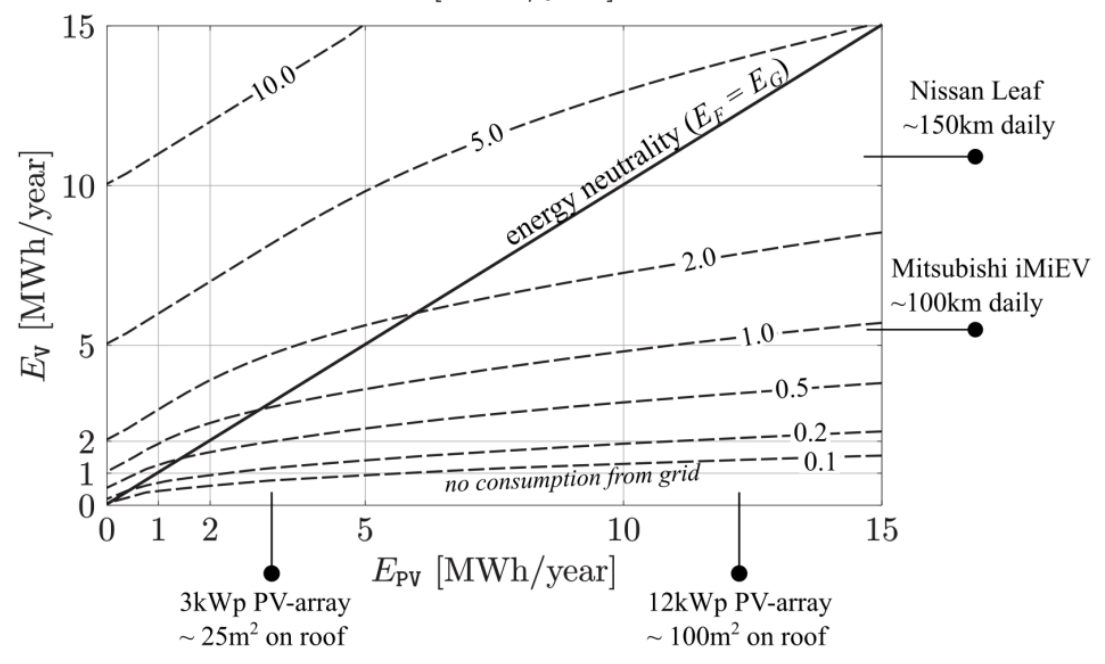

Fig. 8. Consumption of energy from the grid (annually).

The case of Nissan Leaf is more demanding, since the energy-neutrality would require PV-array of nearly $11 \mathrm{kWp}$ (area of $90 \mathrm{~m}^{2}$ ) and grid transfers of $\pm 4 \mathrm{MWh}$ annually. Smaller PVs would not be recommended due to high energy purchase. On the other hand, 
an oversized PV-array, for example $15 \mathrm{kWp}$, could limit the purchase to $3 \mathrm{MWh}$, but at the cost of rising the energy feed to about $7 \mathrm{MWh}$ annually.

\section{Conclusions}

The transition from combustion engine to electric propulsion in transportation seems to be inevitable in the coming decades with view of recent technological advances in this field. However, the energy needed for electric transport is not to be found in the present capability of the utility network. The solution is to harvest the energy from RES.

This paper investigates the benefits and drawbacks of a use-case scenario with one electric car and a dedicated PV-system, suitable for single family usage, in Polish climatic conditions.. The focus is on the idea is that energy for transportation purpose should be produced locally and grid energy transfers should be minimized. The presented method of finding energy-neutrality is universal and applicable for any climate, but the quantitative results in this paper have been calculated for Polish full-year solar irradiance data.

It was found, that grid energy-neutrality of the system can be achieved when annual energy production from PV-system matches the annual EV consumption, regardless the irregularities of daily irradiance or charging profiles. Moreover, the under- or oversizing of PV-arrays would not significantly improve the energy coverage for charging, while having the drawback of greatly increased grid energy transfers.

The overall conclusion is that common use of electric cars - with support of a properly chosen dedicated PV-systems as main source of energy and local energy buffering - would greatly mitigate a threat to the existing utility system.

\section{References}

1. Polska Organizacja Przemysłu i Handlu Naftowego, http://www.popihn.pl

2. Polska Organizacja Gazu Płynnego, http://www.pogp.pl

3. Urząd Regulacji Energetyki, http://www.ure.gov.pl

4. M.S. ElNozahy, M.M.A. Salama, J. Renew. Sustain. Ener. 5 (2013)

5. D.B. Richardson, Renew. Sust. Energ. Rev. 19, 247 (2013)

6. X. Li, L. Lopes, S. Williamson, IEEE Pow. Ener. Soc. Ge. (2009)

7. M.S. ElNozahy, M.M.A. Salama, Electr. Pow. Syst. Res. 110, 133 (2014)

8. W. Marańda, MIXDES (2017)

9. Joint Research Centre Institute for Energy, http://re.jrc.ec.europa.eu/pvgis/

10. W. Marańda, M. Piotrowicz, Int. J. Microelect. Comp. Sci. 5, 92 (2014) 